\title{
WIDE-BAND SPECTRA OF PROMPT EMISSION
}

\author{
K. Asano ${ }^{1}$
}

\begin{abstract}
The Fermi observatory has detected GeV photons from several GRBs. Some of them show an extra spectral component, which is a hint for emission mechanism of GRBs. Here we discuss the GeV emission mechanism according to several promising models. The difference between models will appear in temporal evolution of the photon spectrum. To verify this, instruments that can obtain high photon statistics like CTA are desired. We also discuss the photosphere model, which is recently supported by wide-band observations with Fermi.
\end{abstract}

\section{Introduction}

The typical spectrum of GRB prompt emission is described by the "Band function", which has a peak at $0.1-1 \mathrm{MeV}$ in the $\nu \mathrm{F}_{\nu}$-diagram. Below and above this peak energy $\varepsilon_{\mathrm{p}}$, the spectrum is well fitted by power-law functions with photon indices $\alpha$ and $\beta$, respectively. This spectral shape implies that most of energy is emitted in $0.1-1 \mathrm{MeV}$ range. In the standard internal shock model, shockaccelerated electrons are injected in a relativistically expanding region. The energy distribution of the accelerated electrons at the injection is a power-law shape with a minimum energy $\gamma_{\mathrm{m}} \mathrm{mc}^{2}$. Those electrons emit synchrotron photons, and the peak energy $\varepsilon_{\mathrm{p}}$ corresponds to the typical synchrotron-photon energy emitted from the minimum-energy electrons. However, the physical mechanism that determines $\gamma_{m}$ is not revealed yet. It is also strange that the peak energies in a burst do not show intense variance in most cases. The peak energies seem stable and tend to distribute in a narrow energy range. Since the cooling timescale is much shorter than the dynamical timescale, cooled electrons will distribute below $\gamma_{\mathrm{m}} \mathrm{m}_{\mathrm{e}} \mathrm{c}^{2}$. As a result, this model predicts $\alpha=-1.5$, but the typical observed photon index is -1 .

An alternative model is the photosphere model. Relativistic outflows driven by radiation pressure become optically thin at a certain radius. Thermal photons

1 Interactive Research Center of Science, Tokyo Institute of Technology, 2-12-1 Ookayama Meguro-ku, Tokyo 152-8550, Japan 
from this photosphere are responsible for the spectral peak in this model. So $\varepsilon_{\mathrm{p}}$, which corresponds to the photon temperature at the photosphere, becomes less sensitive to model parameters. This agrees with the observed stability of the peak energy. To reconcile this model with the observed spectra, extra photon production above and below $\varepsilon_{\mathrm{p}}$ is needed in addition to the Planck-like thermal spectrum.

The simple Band function does not bring us so rich information on the unknown emission mechanism of GRBs. Wider-band spectra, such as GeV gamma-rays or optical photons, may give us some clue for the emission mechanism. Historically, the first photon detection other than $\mathrm{keV}-\mathrm{MeV}$ band during the prompt phase is the optical emission from GRB 990123 (Akerlof et al. 1999). This was interpreted as the reverse shock emission due to interaction between the outflow and interstellar medium. However, considering the discovery of the shallow decay phase of the afterglow in the "Swift era", the reverse shock emission may be not ubiquitous. The optical emission in GRB 990123 could have the same origin as the prompt gamma-ray emission. This possibility has been reinforced by subsequent optical detections, such as GRB 041219 or GRB 080319B (Racusin et al. 2008), in which optical variations seem to correlate with the gamma-ray fluxes. In such famous examples, the optical fluxes exceed the extrapolation from the Band function in $\mathrm{X}$-ray range. One possible interpretation is that the optical photons are emitted via synchrotron emission and the gamma-ray emission is due to SSC (synchrotron self-Compton). Another possibility is hadronic models. Asano et al. (2010) interpreted that the optical emission in GRB 080319B is synchrotron emission from secondary electron-positron pairs produced via hadronic cascade, which is initiated by photopion production from accelerated protons.

Interestingly, both the SSC and hadronic models in GRB 080319B predict GeV emission via second-order SSC and the hadronic cascade, respectively. The simultaneous observation from optical to $\mathrm{GeV}$ is desired to determine the model of the optical components, which may constrain the emission mechanisms for the main component as well. But, unfortunately, such an example is not appeared yet. In this paper, we discuss deviations from the simple Band function mainly based on observations with the Fermi observatory.

\section{Fermi 2008-2009}

The Fermi gamma-ray observatory was successfully launched 2008, and have detected several fantastic GRBs accompanying bright GeV emission Some of them show an extra spectral component in GeV band. In the case of short GRB 090510 (Ackermann et al. 2010), a spectral excess around $10 \mathrm{keV}$ is also reported. This soft excess is consistent with the power-law extrapolation from the $\mathrm{GeV}$ extra component. If the magnetic field is relatively low, a simple SSC model can make a $\mathrm{GeV}$ excess. But the low-energy excess seems difficult to be reproduced by SSC models. Nevertheless, time-dependent simulations with very low magnetic field show that remnant electrons emit $10 \mathrm{keV}$ synchrotron photons after the electron injection ends (Asano \& Mészáros 2011). 
Another remarkable feature the Fermi found is delayed onset of $\mathrm{GeV}$ emission relative to $\mathrm{MeV}$ gamma-rays. In GRB 090510, the first intense $\mathrm{MeV}$ pulse of $0.1 \mathrm{sec}$ timescale does not have significant signals in LAT, while the second MeV pulse synchronizes a LAT pulse. In a long GRB, 080916C, the LAT lightcurve shows a $\sim 4$ sec delay in its onset relative to the GBM lightcurve (Abdo et al. 2009). The delayed $\mathrm{GeV}$ onsets arouse possibility that the $\mathrm{GeV}$ emission is due to an early onsets of afterglow (Ghisellini et al. 2010; Kumar \& Barniol Duran 2010) If the initial Lorentz factor is as large as 1000, the onset of the kinetic energy dissipation due to the external shock becomes early. So the afterglow emission can start before the prompt emission ends. In this scenario, the $\mathrm{GeV}$ extra component is explained by synchrotron emission from the external shock. This is consistent with the observed long-lived GeV emission. However, the LAT-lightcurve in GRB 090926A show an intense spike, which seems to coincide with a $\mathrm{MeV}$ spike (Ackermann et al. 2011). In addition, Maxham et al. 2011 claim that the energy-injection rate the GBM lightcurve suggests is not enough to explain the GeV flux in the prompt phase. Thus, the origin of the early $\mathrm{GeV}$ emission is likely internal rather than external.

Two zone models have been proposed to explain both the extra spectral component and delayed onset in GeV range (Toma et al. 2009, 2011; Zou et al. 2011). Emission originated from an inner radius, such as the photospheric emission, constitutes the main Band component. A cocoon surrounding the jet can emit soft photons as well. An energy dissipation of the outflow occurs and electrons are accelerated in this outer region. Those electrons up-scatter photons from the inside regions, which is responsible for the $\mathrm{GeV}$ emission. The geometrical configuration in the two models, namely the spatial separation between the source of the soft photons and the site of the dissipation region, can cause the delayed arrival of the up-scattered soft photons. In such two-zone models, the photons coming from behind tend to be scattered backward in the outer shell frame. This enhances the flux from the off-axis region. Asano \& Mészáros (2011) show that the GeV lightcurve in such two-zone models tends to have a long tail, which is due to the contribution of the off-axis emission

However, as seen in GRB 090217A (Ackermann et al. 2010b), not all LAT-GRBs show delayed onset of GeV emission. So we may not need to persist in the afterglow models or two-zone models for the $\mathrm{GeV}$ emission. Another possible option is emission initiated by the hadronic cascade. Accelerated protons collide with photons and produce pions. The neutral pions decay into high-energy gamma-rays, while charged pions emit synchrotron photons before they decay into muons. Most of such high-energy photons are absorbed via $\gamma \gamma$-pair production. Secondary electron-positron pairs emit photons via synchrotron or inverse Compton (IC) processes. If we adopt the hadronic model to GRB 090510, the required proton energy much exceeds the observed gamma-ray energy (Asano et al. 2009). In this case, the extra component has a hard spectrum with a photon index -1.6 so that IC emission should be prominent to model the extra component by the hadronic cascade. Therefore, the magnetic field becomes very weak, which lowers the efficiency of photopion production. As a result, the required proton 


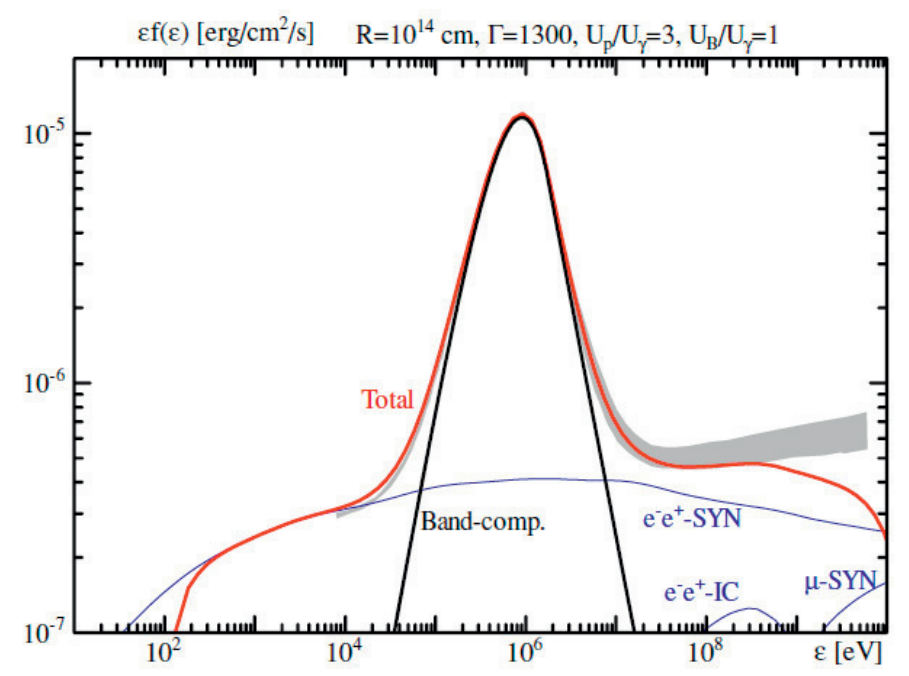

Fig. 1. Spectral fit by a hadronic model for GRB 090902B (Asano et al. 2010, ApJ, 725, L121).

luminosity is more than 100 times the gamma-ray luminosity. If the ultra-high energy cosmic rays (UHECR) above $10^{20} \mathrm{eV}$ are mainly produced from GRBs, proton dominance in energy budget is required. The neutrino limit from GRBs with IceCube (Abbasi et al. 2012) seems severe for the UHECR-production scenario with GRBs, as claimed in Zhang and Kumar 2012. However, the neutrino flux largely depends on the model parameters such as Lorentz factor or emission radius (see e.g. Gao et al. 2012, and references therein). Considering the variety in parameters, the hadronic model seems still viable.

More plausible case for the hadronic model is GRB 090902B (Abdo et al. 2009b). In this case, the extra component has a flat spectrum, which dominates $\mathrm{GeV}$ and $10 \mathrm{keV}$ regions. An IC component is not needed so that we can assume a strong magnetic field. Consequently, the required proton energy is comparable to the gamma-ray energy in $\mathrm{MeV}$ region (Asano et al. 2010, see Fig. 1).

Time-dependent simulations by Asano \& Mészáros (2012) show that the hadronic GeV emission has a broader lightcurve than leptonic emission, and tends to delay relative to the $\mathrm{MeV}$ lightcurve (see Fig. 2). This tendency is due to the long acceleration timescale of protons and the continuous photopion production after the end of the particle injection.

In summary, each model has its own characteristic in the GeV lightcurve. The afterglow models predict smooth and featureless lightcurve, while GeV emission of internal origin may have a strong variability correlating with the $\mathrm{MeV}$ lightcurve. A long-tail in a $\mathrm{GeV}$ pulse may imply the two zone models with external Compton scattering. If we can statistically investigate the pulse onset 


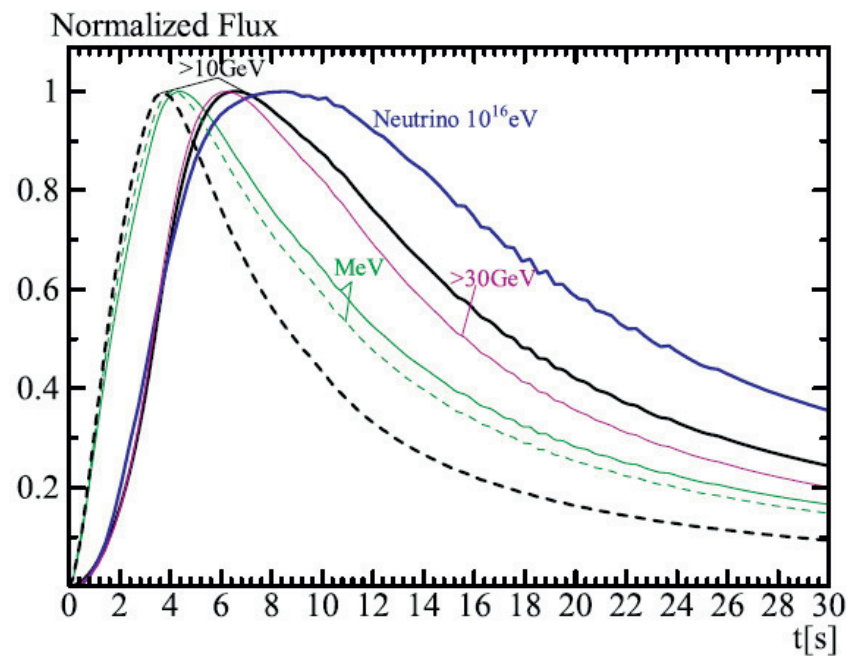

Fig. 2. Lightcurves for hadronic (solid) and leptonic (dashed) models (Asano \& Mészáros 2012, ApJ, 757, 115).

and profile for $\mathrm{GeV}$ and $\mathrm{MeV}$ emissions, the difference in leptonic and hadronic models may be clarified.

Unfortunately, the effective area of the Fermi-LAT is not sufficient to distinguish the models. A future project of the air Cherenkov telescopes, CTA, will try to lower the energy threshold as low as $\sim 30 \mathrm{GeV}$. The expected detection rate of GRBs with CTA is not so high. But if CTA detects a GRB, its high photon statistics bring us clue to determining the origin of the $\mathrm{GeV}$ emission (Inoue et al. 2012).

\section{Fermi 2010-}

Recently, the Fermi-LAT has not detected so intense GRBs. As the number of the GRB samples increases, the Fermi-LAT reveals that the extra-component in $\mathrm{GeV}$ range is not common for all GRBs (Ackermann 2012). The GRBs with extra components may belong to a particular group. However, by fitting the spectrum for a wide energy range, certain deviations from the Band function below $\varepsilon_{\mathrm{p}}$ have been found. Those spectra are said to be consistent with a superposition of the Band function and Planck spectrum.

Some authors claim that this implies the photospheric emission. For example, the time-resolved emission spectrum for GRB 110721A is well fitted by the Band function with an additional blackbody component (Axelsson et al. 2012). In this GRB, the initial peak energy $\varepsilon_{\mathrm{p}} \sim 15 \mathrm{MeV}$ is the highest ever detected. The low-energy spectral index is about -1 , which is hard to be explained by the usual synchrotron model as we mentioned in Introduction. The dissipative photosphere models may explain this spectral shape. Some dissipation mechanism around the 


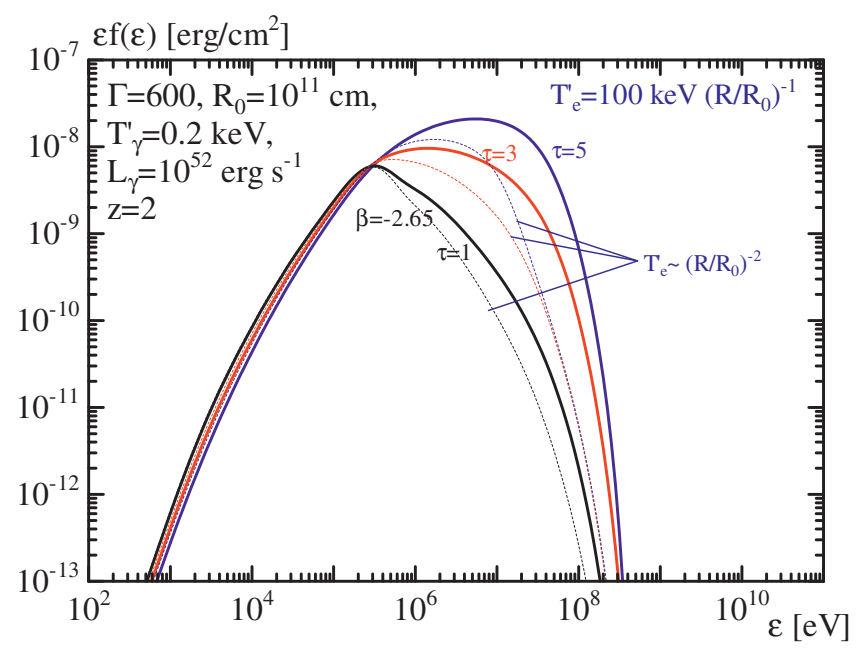

Fig. 3. Optical depth dependence in dissipative photosphere models. The electron temperature drops with proportional to $\mathrm{R}^{-1}$ (solid) and $\mathrm{R}^{-2}$ (dotted) (Asano \& Mészáros in prep.).

photosphere heats electrons. Compton scattering by heated electrons may produce the Band-like spectrum with a $15 \mathrm{MeV}$ peak.

However, the detected deviations from the Band function do not necessarily mean existence of the photospheric emission. Those spectra may be fitted with two Band functions. To begin with, it is not verified that the Band function is universal for the prompt emission. No model is assured to reproduce the Band spectrum so far. While the dissipative photosphere models are attractive, there is great uncertainty in the spectral shape. Beloborodov (2010) demonstrates the Band spectrum by a dissipation mechanism initiated by proton-neutron collisions. However, the spectral shape is sensitive to the model parameters. As shown in Figure 3, the parameter range that agrees with the Band function is very limited (Asano \& Mészáros in prep.). In this calculation, the dissipation starts at $R=R_{0}$, and electron temperature is assumed to be evolved as $T=100 \mathrm{keV}$ $\left(R / R_{0}\right)^{-1}$ or $\left(R / R_{0}\right)^{-2}$. If the initial optical depth is larger than unity, photons are efficiently up-scattered above $\mathrm{MeV}$. However, the resultant spectrum has a curved shape, which may be not consistent with the combination of the Band function and blackbody spectrum as claimed in GRB 110721A On the other hand, a low initial optical depth results in poor efficiency to produce high-energy photons above $\varepsilon_{\mathrm{p}}$. To verify the spectral curvature, we may need better photon statistics in $1-10 \mathrm{MeV}$ energy range.

\section{Hint on emission mechanism}

As mentioned above, the most frequently discussed model to resolve the problem in the hard low-energy spectrum is the dissipative photosphere The narrow Band 


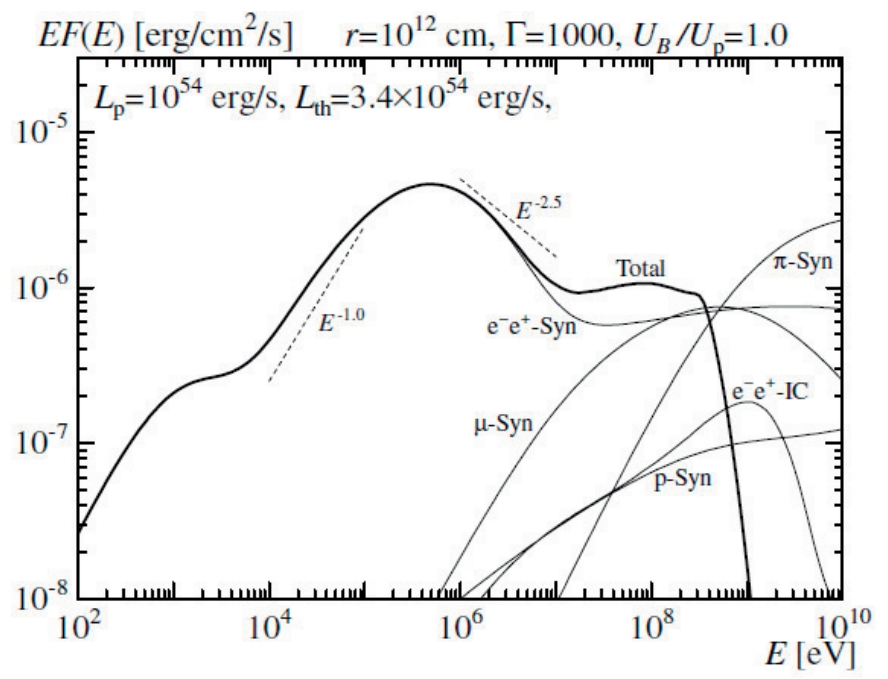

Fig. 4. Calculated spectrum for a turbulence heating/acceleration model with pp-induced hadronic cascade (Murase et al. 2012).

component in GRB 090902B (see Fig. 1) is an encouraging example for this model. However, the several detections of strong polarization (e.g. Yonetoku et al. 2012) are not advantageous for the photosphere model. The small radius and large Lorentz factor supposed in this model suggest a very short variability. But the typical variability timescale is $\sim 0.1 \mathrm{sec}$. Therefore, the timescale of the engine activity should be so long that the outflow can be treated as a steady flow compared to the dynamical timescale at the photosphere.

Another model we recommend is continuous electron heating by turbulences. An internal shock due to a collision of two inhomogeneous shells can induce magnetohydrodynamical turbulence via the Richtmyer-Meshkov instability (Inoue et al. 2011). Such long-lived turbulences may heat/accelerate electrons via a similar process to the second-order Fermi acceleration. If this heating mechanism is effective, the balance between electron heating and cooling determines the typical energy of electrons. The synchrotron photon energy from the balanced electrons corresponds to the spectral peak energy. The resultant electron spectrum becomes hard, and the photon spectrum can be consistent with the low-energy spectral index (Asano \& Terasawa 2009) Murase et al. (2012) demonstrate that a combination of this turbulent acceleration and high-energy electron injection due to pp-collision induced hadronic cascade at the photosphere can reproduce the Band function with low and high-energy extra components. In this model, the hadronic cascade can inject electrons in a high-energy region at which turbulence acceleration does not work because of synchrotron cooling. So the hard spectrum above $\varepsilon_{\mathrm{p}}$ and extra component are reproduced (see Fig. 4). 
At present we do not have a convincing model to explain the Band spectrum yet. Each model has its advantages and disadvantages. In order to reveal the emission mechanism, future wide-band observations including temporal evolution will be indispensable.

I thank my collaborators, P. Mészáros, T. Terasawa, S. Inoue, K. Murase, S. Guiriec, T. Inoue, K. Ioka, and S. Gao. I am supported by Grants-in-Aid for Scientific Research No. 22740117 from the Ministry of Education, Culture, Sports, Science and Technology (MEXT) of Japan.

\section{References}

Abdo, A.A., Ackermann, M., Arimoto, M., et al., 2009, Science, 323, 1688

Abdo, A.A., Ackermann, M., Ajello, M., et al., 2009b, ApJ, 706, L138

Abbasi, R., Abdou, Y., Abu-Zayyad, T., et al., 2012, Nature, 484, 351

Ackermann, M., Asano, K., Atwood, W.B., et al., 2010, ApJ, 716, 1178

Ackermann, M. Ajello, M., Baldini, L., et al., 2010b, ApJ, 717, L127

Ackermann, M. Ajello, M., Asano, K., et al., 2011, ApJ, 729, 114

Ackermann, M., Ajello, M., Baldini, L., et al., 2012, ApJ, 754, 121

Akerlof, C., Balsano, R. Barthelmy, S., et al., 1999, Nature, 398, 400

Asano, K., Guiriec, S., \& Mészáros, P., 2009, ApJ, 705, L191

Asano, K., Inoue, S., \& Mészáros, P., 2010, ApJ, 725, L121

Asano, K., \& Mészáros, P., 2011, ApJ, 739, 103

Asano, K., \& Mészáros, P., 2012, ApJ, 757, 115

Asano, K., \& Mészáros, P., in preparation

Asano, K., \& Terasawa, T., 2009, ApJ, 705, 1714

Axelsson, M., Baldini, L., Barbiellini, G., et al., 2012, ApJ, 757, L31

Beloborodov, A.M., 2010, MNRAS, 407, 1033

Gao, S., Asano, K., \& Mészáros, P., 2012, JCAP, 058

Ghisellini, G., Ghirlanda, G., Nava, L., \& Celotti, A., 2010, MNRAS, 403, 926

Inoue, S., Granot, J., O'Brien, P.T., et al. (for the CTA Consortium), 2013, Astrop.

Phys., accepted

Inoue, T., Asano, K., \& Ioka, K., ApJ, 734, 77

Kumar, P., \& Barniol Duran, R., 2010, MNRAS, 409, 226

Maxham, A., Zhang, B.B., \& Zhang, B., 2011, MNRAS, 415, 77

Murase, K., Asano, K., Terasawa, T., \& Mészáros, P., 2012, ApJ, 746, 164

Racusin, J.L., Karpov, S.V., Sokolowski, M., et al., 2008, Nature, 455, 183

Toma, K.,Wu, X.-F., \& Mészáros, P., 2009, ApJ, 707, 1404

Toma, K.,Wu, X.-F., \& Mészáros, P., 2011, MNRAS, 415, 1663

Yonetoku, D., Murakami, T., Gunji, S., et al., 2012, ApJ, 758, L1

Zhang, B., \& Kumar, P., 2012 [arXiv:1210.0647]

Zou, Y.-C., Fan, Y.-Z., \& Piran, T., 2011, ApJ, 726, 2 